\title{
Stylistique et discours scientifique
}

\section{Gilles Mathis}

\section{OpenEdition}

Journals

Édition électronique

URL : https://journals.openedition.org/asp/3020

DOI : 10.4000/asp.3020

ISSN : 2108-6354

\section{Éditeur}

Groupe d'étude et de recherche en anglais de spécialité

\section{Édition imprimée}

Date de publication : 1 décembre 1997

Pagination : 157-183

ISSN : 1246-8185

\section{Référence électronique}

Gilles Mathis, «Stylistique et discours scientifique », ASp [En ligne], 15-18| 1997, mis en ligne le 25 mai 2012, consulté le 21 septembre 2021. URL : http://journals.openedition.org/asp/3020 ; DOl : https:// doi.org/10.4000/asp.3020

Ce document a été généré automatiquement le 21 septembre 2021.

Tous droits réservés 


\title{
Stylistique et discours scientifique
}

\author{
Gilles Mathis
}

\section{Introduction}

1 Le littéraire que je suis et qui se pose depuis près de trente ans la question de savoir «Quel discours scientifique pour la stylistique?» n'aurait jamais soupçonné qu'il lui faudrait un jour renverser cette question et s'interroger sur «Quelle stylistique pour le discours scientifique?». Je commencerai donc par remercier Michel Petit pour cette initiative originale qui nous rappelle fort opportunément que, parmi les pratiques discursives, la littérature ne peut confisquer la stylistique et j'enchaînerai par un aveu : je crains en effet que mon intervention ne frôle l'imposture car je prétends parler d'un domaine (le discours scientifique) que je ne maitrise pas et d'une problématique dont j'ignore tout ou presque, ayant trop longtemps sans doute écouté d'autres sirènes et le temps m'ayant manqué pour le minimum de recyclage que supposait l'entreprise. Je parlerai donc sous le triple contrôle des linguistes, des spécialistes de l'anglais de spécialité et bien sûr des scientifiques de cette assemblée. Cette crainte se double par ailleurs d'une certaine gêne, dans la mesure où mon propos est placé sous le signe d'un autre paradoxe. Une certaine éthique ou un certain sens de l'esthétique (du décorum, disaient les Anciens) voudrait en effet que mon propos se rapprochât autant que possible de son objet d'application et se fit aussi «scientifique » que possible. Or la stylistique n'est pas une science mais une pratique ou plutôt un ensemble de pratiques irrémédiablement marqué, à des degrés divers et singulièrement en "stylistique littéraire ", par un subjectivisme qu'il est, soit dit en passant, préférable d'assumer plutôt que de tenter de nier. Toutefois, dans la mesure où la stylistique, bien comprise, tente d'importer dans son domaine le souci de l'observation minutieuse, l'esprit de rigueur, la concision et la précision du langage qui marquent, entre autres qualités, le discours scientifique, elle reste, sinon une «science» du moins une discipline, dans tous les sens du terme. À ce titre, elle a peut-être droit de cité (d'être citée) dans le domaine qui nous occupe aujourd'hui. 
2 Après quelques réflexions préliminaires sur l'intitulé du Colloque, suivies d'un rappel schématique de la spécificité du « discours scientifique » et des pratiques stylisticiennes, je tenterai de répondre à la question posée, sans trop d'illusions sur l'originalité des réponses. En annexe, figurera un corpus très restreint de quelques exemples (tirés des mathématiques et de la physique) suivis de quelques brefs commentaires stylistiques. Mon discours sera surtout général, plus spéculatif peut-être que théorique, et aussi limité que le corpus.

3 La réflexion n'abordera pas les aspects historiques de la problématique, c'est-à-dire l'émergence et l'évolution du discours scientifique de l'Antiquité à nos jours. Rappelons seulement que la distinction des domaines et des variétés de langue est une conquête progressive et la caractéristique de la pensée moderne. Une pensée qui, malgré les indéniables progrès accomplis au cours des siècles, n'en finit pourtant pas de s'interroger sur le problème des frontières entre les disciplines et la validité des théorisations. On sait que, à l'origine (?), philosophie, littérature (voire poésie) et science sont étroitement liées. Platon était mathématicien et il avait inscrit au fronton de son Académie l'avertissement suivant: "Que nul n'entre ici s'il n'est géomètre ». Pourtant, l'on sait aussi la part que joue l'imaginaire (et notamment les mythes) dans son discours ${ }^{1}$. D'autre part, nombre de traités scientifiques (travaux des champs ou autres calendriers de la vie pratique) et de traités juridiques étaient écrits en vers, pour des raisons mnémotechniques ${ }^{2}$. La genèse de la pensée scientifique est l'histoire de sa libération progressive de l'emprise de l'imaginaire sous toutes ses formes: superstitions, mythes, croyances.

4 Je n'aborderai pas non plus le problème important et passionnant de la traduction des discours scientifiques d'une langue à l'autre, domaine dans lequel mes ignorances sont peut-être encore plus abyssales, ni bien sûr le fonctionnement du " discours scientifique " inséré dans un texte littéraire, qui relève d'une autre problématique que celle de ce Colloque.

\section{Les présupposés derrière cette question «Quelle stylistique pour le discours scientifique? »}

5 Le titre du Colloque suggère au moins deux présupposés: le premier est clair, « il y a plusieurs stylistiques», le second, «il y a un discours scientifique», est plus problématique ; tout dépend du sens donné à l'article défini et à l'expression « le discours scientifique ».

6 Je ne pense pas qu'il y ait lieu de s'attarder sur le premier. Le problème de la pluralité des pratiques n'est pas propre à la stylistique. Il est au cœur même de toutes les activités humaines. Comme je viens de le faire, l'emploi du singulier «la stylistique » (comme la linguistique, la philosophie, la science, etc.) n'est qu'une commodité (une figure de style? ) qui désigne un certain type d'activité ou un champ d'investigation, tandis que le pluriel renvoie à la diversité des démarches selon l'objectif poursuivi et l'objet d'analyse (voir infra). Le Colloque pose donc la question de savoir quelle est la pratique stylisticienne la mieux adaptée à l'étude du « discours scientifique »?

7 Le second présupposé, «il y a un discours scientifique », est peut-être innocemment et faussement provocateur. Si l'on considère que «discours » et «langage » sont ici synonymes, on peut objecter que l'autonomie du discours scientifique, entendu ici 
comme discours tenu par un spécialiste des sciences exactes ou expérimentales portant sur des objets de connaissance relevant de sa discipline, n'a jamais été "scientifiquement» démontrée, mais l'objection vaut pour les autres discours, par exemple, littéraire ou poétique, pour évoquer l'autre pôle des productions langagières, car le délicat problème des frontières entre les domaines, est loin d'être résolu. Pourtant cette lacune théorique n'a jamais empêché la recherche et la critique de se poursuivre et, pour l'instant, en l'absence d'une véritable typologie des discours, qui n'est peut-être qu'un mythe, linguistes, stylisticiens et littéraires, tous logés à la même enseigne, doivent se contenter de la connaissance empirique - certes, de plus en plus affinée au cours des siècles - qu'ils possèdent des différences d'un domaine à l'autre. Dans l'écrasante majorité des cas, la compétence linguistique de l'auditeur/lecteur et la situation d'énonciation lui permettent en effet de savoir s'il a affaire à un texte scientifique ou non. Il n'y a donc aucun inconvénient à parler de « langage scientifique » comme on parle de "langage poétique», étant bien entendu que, dans les deux cas, le mot «langage", comme « langue " ${ }^{3}$, est une abstraction, qui recouvre une grande diversité de productions discursives qui ont cependant quelques traits (qualités ou propriétés) en commun, un " air de famille » si l'on veut, qui permettent de les classer dans une même variétét de langue (voir infra).

8 Cela dit, toutes les langues de spécialité ne peuvent être mises sur le même rang. Certaines s'écartent plus que d'autres du langage courant, notamment au plan lexical. Qui songerait à nier que la mathématique (ou les mathématiques), même si elle emprunte une partie de son lexique à la langue courante, constitue un langage original, fortement technique et spécialisé, parfois incompréhensible pour le profane, et pas seulement en raison de son haut degré d'abstraction? À tel point que l'enseignement des mathématiques passe d'abord par l'apprentissage d'un certain langage, comme on l'oublie trop souvent: la première tâche à laquelle est confronté l'écolier dès son plus jeune âge est sans doute moins la maitrise du calcul (au sens large du terme) que le décodage de l'énoncé mathématique et le maniement d'un idiome.

9 Toutefois dans notre société, dans notre monde devrais-je dire, en pleine mutation depuis l'explosion de la recherche scientifique avec la conquête de l'atome et de l'espace et le développement de l'informatique, sans parler de la mondialisation de l'économie et de la politique, les rapports entre langue commune ou «standard» et langues de spécialités ont changé. L'écart à la fois se comble et se creuse. Il se creuse parce que le lexique spécialisé ne cesse de s'enrichir de mots nouveaux à chaque découverte et qu'il est difficile de suivre les progrès fulgurants de la science dans certains secteurs de pointe; il se comble parce que l'intégration de ces néologismes scientifiques dans la langue courante est de plus en rapide en raison des immenses progrès en matière de communication réalisés depuis le développement des media, notamment de la télévision, et plus récemment de l'informatique qui ouvre dans le domaine de l'acquisition des connaissances des perspectives vertigineuses. En raison aussi de ce que l'on pourrait appeler la domestication de la science, c'est-à-dire la multiplication des applications pratiques des découvertes dans la vie quotidienne, la cuisine devient un mini-laboratoire et la chambre ou le bureau un centre multi-média où trône l'ordinateur, cette nouvelle idole des jeunes... de tous âges. Le succès de quantités de revues informatiques spécialement conçues pour les «nuls $»^{5}$ et qui envahissent les consoles des grands centres de distribution montre assez que le recyclage "scientifique » de l'homme moyen est une nécessité vitale : l'homme du troisième millénaire sera un savant ou ne sera rien. Aussi la 
langue de tous les jours se transforme-t-elle sous la pression, chaque jour plus forte, de certaines langues de spécialité, et son lexique devient de plus en plus scientifique, dans son contenu comme dans sa forme: à côté des "technicismes", les anglicismes fleurissent dans la langue française, parce que, n'est-ce pas, on (les responsables des media) n'a plus le temps de traduire (le temps c'est de l'argent) tandis que se multiplient les sigles (analogues linguistiques des symboles mathématiques), si bien que la lecture devient parfois un véritable jeu de pistes ou un parcours d'initié6. Bref la langue "commune " le devient de moins en moins et si l'éducation (la masse) ne suit pas, les conséquences à plus ou moins long terme sur le plan sociologique (y compris les relations parents/enfants) risquent d'être catastrophiques ${ }^{7}$. Mais ceci est une autre histoire.

\section{De la stylistique et de ses pratiques}

1. La stylistique ${ }^{8}$ est au carrefour de nombreuses disciplines comme la linguistique, la philologie, la psychologie, la sociolinguistique, l'histoire des mentalités, la critique littéraire, l'esthétique et son histoire, la pragmatique, la sémiotique (narrative, poétique ou dramatique), mais elle reste une discipline autonome, avec ses propres objectifs, sa terminologie, ses protocoles d'analyse ${ }^{9}$.

2. La notion de "style ", même entendue comme " différence ", ou " manière originale ", ou « ensemble d'habitudes de composition » reste sinon ambiguë du moins ambivalente parce que paradoxale et appliquée à différents niveaux d'analyse qui peuvent ou non se recouper: texte isolé, genre, œuvre, individu, groupe d'auteurs, période historique, nation tout entière.

Le stylisticien doit donc se faire une règle absolue de préciser le sens qu'il prête à ce terme, au seuil de ses analyses. En critique littéraire, par exemple, les définitions oscillent entre deux extrêmes, le style réduit à une simple empreinte ou purement et simplement identifié au texte tout entier (considéré comme, singulier, unique), en passant par le style comme ornement (ou addition), comme forme (et stratégie de la forme), comme sens ${ }^{10}$ (ou vision du monde), comme choix, comme connotation, etc.

3. Le style individuel est plus ou moins marqué, plus ou moins caché. Alors qu'il doit en principe frapper comme une évidence (le style existe pour être perçu, parce qu'il est perçu), il évolue souvent aux franges du «dire», dans les zones instables de la signification, dans les connotations, l'implicite, le non-dit, l'insaisissable. Il faut donc, sans doute, postuler l'existence de niveaux du style : une face manifeste, avec ses traits de style bien marqués, comme des billets de banque, rendant possible l'imitation, mais aussi une face cachée, intime, inviolable, qui fait que la contrefaçon restera toujours une contrefaçon, une réussite provisoire condamnée à l'échec. Un cliché du genre (du style !) : «Il/elle n'a pas créé de style; il/elle avait un talent inimitable» résume bien les paradoxes et ambiguïtés du langage : selon les perspectives, « style ", « talent », " génie » se confondent ou s'opposent.

4. Si le style, dans son acception peut-être la plus répandue de "manière personnelle ", de «marque individuelle», est, en théorie, la différence absolue (mais comment l'établir, puisque la production est continue et infinie ${ }^{11}$ ?), l'observation montre que la notion de "style » est souvent relative et plurielle, car un style individuel évolue. Bien souvent, lorsqu'on parle du «style» d'un auteur, il s'agit du «style de la maturité » (terme 
ambigu) ou de ses "meilleures œuvres", corpus établi par consensus, tributaire de valeurs historiquement datées, de l'idéologie régnante et de modes esthétiques.

5. Le texte (qu'il soit oral ou écrit) existe comme « objet » d'analyse. Même s'il est en partie construit par l'analyse, le texte possède son vocabulaire, sa syntaxe, son rythme, bref une forme que l'on peut décrire et cette forme produit des effets que l'on peut repérer et analyser. Le stylisticien n'ignore pas la problématique de l'objet et du sujet, mais ce n'est pas sa priorité. Son approche du texte reste globalement structuraliste et pragmatique.

6. La stylistique est avant tout une pratique descriptive. Elle commence par la collecte des faits " pertinents $»^{12}$ aux différents niveaux de l'expression (lexique, syntaxe, phonologie, rythme et prosodie). Proche de la description linguistique à laquelle elle emprunte souvent terminologie, modèles et protocoles d'analyse, elle en diffère par ses finalités ${ }^{13}$, ses méthodes d'analyse textuelle, éventuellement le choix du corpus, mais il faut reconnaître que ces différences ont commencé à se réduire avec l'élargissement de la linguistique au domaine du discours, y compris littéraire ${ }^{14}$. Précisons que le stylisticien n'est pas dupe du caractère faussement scientifique du mot « descriptif » qui n'est pas si aisément séparable de "interprétatif ", son frère (apparemment) ennemi. En effet, dans la mesure où aucune analyse stylistique ne saurait être exhaustive ${ }^{15}$, elle implique le choix d'un corpus, lequel implique déjà une interprétation, donc l'intervention de critères partiellement subjectifs. Cela dit, si «interprétatif» signifie analyse (description?) du fonctionnement des faits stylistiques en contexte, la stylistique est aussi évidemment interprétative. Quant au jugement de valeur esthétique, il ne relève pas de son domaine.

17 7. La stylistique étudie des différences, des variations, des contrastes, mais aussi des redondances (lesquelles peuvent fonder des ruptures). Par conséquent, les notions de norme (externe ou interne), d'écart et de déviation sont pertinentes. Quelles que soient les objections que soulève la définition théorique de ces concepts, le stylisticien constate chaque jour qu'ils sont incontournables comme le prouve le métalangage de leurs propres détracteurs dès qu'ils passent de la théorie à la pratique.

Qu'il s'agisse de discours oral ou écrit (et la distinction est capitale, en stylistique), on peut distinguer deux grandes pratiques stylisticiennes, selon l'objectif visé, qu'il convient de bien distinguer, auxquelles on peut annexer l'analyse rhétorique.

Ou bien l'on décide de cerner les constantes d'une pratique langagière à différents niveaux d'investigation (auteur, groupe d'auteurs, période, genre, etc.) et c'est l'étude de style ("style study») qui recense les traits de style («traits of style »), comme on dit «traits de caractère ». Il s'agit d'une stylistique de caractérisation (signalétique), à la recherche de traits distinctifs, d'une différence, et sa démarche est essentiellement comparative.

Ou bien, on cherche à rendre compte du fonctionnement d'un texte isolé, à montrer en quoi il est efficace (sur le lecteur) et comment les procédés linguistiques participent à la production du sens et c'est l'analyse stylistique («stylistic analysis») qui recense les faits stylistiques («stylistic devices»). Il s'agit d'une stylistique textuelle ${ }^{16}$ dont le but n'est pas tant de définir la spécificité d'un objet que d'étudier une stratégie de la forme ${ }^{17}$ et de la réception du message.

21 Cette pratique est en rapports étroits avec l'analyse rhétorique, qu'il s'agisse de la rhétorique restreinte (domaine des figures) ou de la rhétorique générale (techniques 
d'argumentation et de persuasion). Pourtant il ne faut pas confondre les deux types d'analyse car si le fait stylistique simple est souvent un fait rhétorique, il déborde largement le cadre de cette discipline qu'on a parfois appelée la "stylistique des Anciens ». On peut donc conserver l'analyse rhétorique dans le domaine de la stylistique (après tout l'expression, ambiguë il est vrai, de "figures de style ", nous y invite) à condition d'éviter la confusion terminologique et de ne pas perdre de vue quelques différences élémentaires ${ }^{18}$.

Les deux concepts opératoires peuvent se recouper s'il est prouvé, par les méthodes comparatives adéquates, que tel « fait stylistique ", qui a d'abord retenu l'attention pour ses effets, se trouve, de surcroît, être original ou particulièrement insistant. Par ailleurs, «trait de style» et «fait stylistique » peuvent être simples ou complexes, de nature substantielle (un procédé concret, repérable à la surface du texte) ou relationnelle (un procédé plus complexe, plus abstrait, de structuration qui suppose la mise en relation de plusieurs faits linguistiques ou textuels [Mathis 1994]. Dans la pratique, la caractérisation des traits de style suppose l'établissement préalable des faits stylistiques. L'analyse stylistique précède donc l'étude de style.

Mais revenons aux "traits de style» en ajoutant une précision. Le recensement des habitudes de composition d'un auteur ne suffit pas à fonder l'originalité d'un style car, je l'ai dit, ce style peut être plus ou moins imitatif ou original. Sa caractérisation peut permettre de situer un auteur dans un genre, dans une période, dans une école. Tel poète sera déclaré lyrique et baroque, par exemple, et c'est une première façon de définir son style. Mais, bien sûr, plus stimulante, et aussi plus ambitieuse, est la recherche de ce qui fait vraiment son originalité au sein de cet ensemble, c'est-à-dire le repérage de traits de style vraiment personnels. L'entreprise est délicate et souvent hasardeuse car, à ce niveau, des facteurs historiques, biographiques, idéologiques et psychologiques entrent en jeu, qui rendent difficile toute formalisation d'un phénomène qui ne peut se réduire à de simples procédés linguistiques, si bien que la célèbre expression, «ce je ne sais quoi », qui définissait jadis le style a encore de beaux jours devant elle, même si les progrès constants de la recherche en la matière autorisent quelques espoirs. Quoi qu'il en soit, ces traits de style sont en général en nombre limité, parfois même réduits à l'unité (la phrase longue de Proust, les imparfaits de Flaubert, la litote classique ou Klassische Dämpfung si bien analysée par Leo Spitzer, etc.). Pour un individu, ils doivent, en principe, se retrouver dans la diversité de ses productions, avec cette réserve que le style peut évoluer, comme dit plus haut. Pour un genre littéraire donné, les traits de style correspondent aux conventions de genre (genre markers) qui permettent de le distinguer des autres genres, par exemple, le discours épique du discours lyrique.

\section{Du discours scientifique : « penser c'est peser » (Lord Kelvin)}

L'expression "discours scientifique ", on l'a dit, fait problème, mais le substantif n'est pas seul en cause. À partir de quelle limite (frontière) passe-t-on du discours non scientifique au discours scientifique, lequel ne peut se définir exclusivement ni par son contenu (objet, vérité), ni par sa forme, ni par son intention, ni par son mode de diffusion, mais par tous ces éléments à la fois, selon un dosage difficile à établir « scientifiquement »? 

trop de sottises, en imaginant un discours scientifique «idéal» dont les mathématiques seraient le modèle le plus approchant, comme le suggère l'étymologie (mathêma = science) ${ }^{19}$ et en nous inspirant partiellement des réflexions de Bally ${ }^{20}$ sur le comportement humain fait, selon le maître de la stylistique affective et expressive de la langue (et non $\mathrm{du}$ discours littéraire), de perception (nous comprenons) et d'émotion (nous sentons), correspondant aux deux pôles intellectuel et affectif entre lesquels la pensée sans cesse oscille.

1. Le discours scientifique est avant tout un discours "sérieux ", au sens que le terme prend dans la théorie des «actes de paroles» (Austin, Searle) et stable. Le ludique, qui tient une place importante en littérature comme dans la langue courante, est banni. Le mimétique aussi car le texte scientifique ${ }^{21}$, en dehors évidemment de ses aspects graphiques, ne vise jamais à imiter son objet à travers sa forme générale et son langage (pas de calligrammes, d'onomatopées, de symbolisme sonore, d'effets de rythmes, etc.).

appartient à la catégorie des textes logiques, hautement formalisé ${ }^{22}$, symbolique et artificiel. C'est d'ailleurs parce qu'il est strictement (rigidement, mécaniquement) codifié qu'il se prête si bien à la parodie. Il suffit de relire le célèbre pastiche de G. Perec ( Cantatrix Sopranica, voir Annexe 11, pour quelques extraits) pour s'en convaincre, en notant qu'il nous en apprend beaucoup sur la nature du discours scientifique (ici science de l'observation) mais aussi sur le langage littéraire, surtout dans les passages les plus facétieux, où les effets sont grossis jusqu'au grotesque. Ainsi la démarche suivie pour la " démonstration » parodique est exemplaire : réfutation de thèses antérieures et nouvelle hypothèse, présentation et conduite de l'expérience (matériel, cobayes, stimuli, méthode, enregistrement, résultats) commentaire. Comme est exemplaire, sans parler du choix de l'anglais, langue universelle (donc idiome privilégié pour la science), la forme hétérogène du discours, mêlant le linguistique (encombré des inévitables parenthèses biographiques, dates à l'appui, comme dans Jean-Page \& Desmeyeurs 1932) et le graphique, sous toutes ses formes (tableau, figures, diagrammes, schémas...).

5. Son langage est rationnel, intellectuel ${ }^{23}$, dénotatif, explicite (pas de non-dit ni d'implicite), univoque (pas de polysémie ni de polyphonie), transparent (bien que le vocabulaire soit souvent opaque pour le profane), libre de toute interférence lexicale (diatopique, diachronique, diastratique, diaphasique ${ }^{24}$ ) dominé par les fonctions référentielle et métalinguistique (définition précise des termes techniques, chaque fois que nécessaire). La fonction poétique, au sens jakobsonien du terme (attention centrée sur la forme du message et non sur le contenu) n'y joue aucun rôle, car l'objectif de la science est la description du réel et la démonstration de la vérité (objective, exacte, comme les sciences du même nom) tandis que la littérature est fiction, imagination, émotion (langue des rêves et des sentiments plutôt que langue des idées), opacité, transposition et nontranscription du réel, fortement marquée par le souci esthétique ${ }^{25}$. Aussi, la littérature est-elle plus souvent mensonge (et songe) que vérité ("pas de vérité du texte», dit Barthes), un «mentir vrai » peut-être, mais cette vérité n'est pas objective (vérité des émotions - peu importe la réalité ou non des stimuli - par opposition à la vérité des faits). 

e sens se construit progressivement, de proposition en proposition, lesquelles sont ordonnées selon une logique argumentative, cumulative et économique, (le superflu, le redondant et le digressif sont bannis), la vérité du discours étant la somme ou le résultat des vérités ${ }^{26}$, partielles de chaque proposition dont aucune ne peut être omise car chaque maillon de la chaîne du raisonnement compte. La démarche heuristique est soit déductive (mathématiques) soit inductive (sciences expérimentales ou d'observation), deux types de raisonnement qu'on oppose habituellement, mais qui se complètent. La science est le domaine du réel quantifiable, mesurable, vérifiable; la littérature, celui de la fiction, de l'impalpable, de la démesure, de l'inqualifiable, même, particulièrement en poésie où le sens est parfois insaisissable, jusqu'au non-sens. Si l'empire des chiffres ne s'en laisse pas conter, dans celui des signes imaginaires, c'est le rêve qui compte.

7. Le texte scientifique est clos, autonome, complet, comme une équation mathématique. Sa vérité est tout entière contenue dans les composants de cette équation et le connecteur logique qui les unit, elle ne se discute pas. Le texte littéraire est clos-ouvert et sa "vérité » (pace Barthes!) dépend moins des faits et des réalités du monde extérieur que des effets produits sur le lecteur, elle est par conséquent éminemment subjective, et l'intertextualité y tient une place prépondérante ; elle est même pour certains le premier critère de "littérarité ». Il y a du citationnel dans le discours scientifique (postulats, théorèmes, etc.) mais c'est un passage obligé, un maillon indispensable dans la chaîne des raisonnements logiques et non plus un argument d'autorité (une caution) ni un hommage en passant aux illustres prédécesseurs.

33 8. Le texte scientifique peut voisiner avec d'autres textes de même nature dans une revue mais il n'entretient en principe aucun rapport avec eux : juxtaposition ne signifie pas intégration. Le texte littéraire, en revanche, peut être un élément dans un ensemble (« sonnet sequences » de Sidney, Spenser ou Shakespeare, par exemple) qui participe à sa signification et valeur esthétique, d'où la pratique de la " mise en série » (en perspective) dans l'analyse stylistique des recueils. Cette "mise en série» est inconcevable ou exceptionnelle dans le domaine scientifique.

9. Malgré sa texture serrée et sa forte unité logique, le texte scientifique n'est pas parfaitement homogène : il est constitué de deux types de discours, linguistique et formel (au sens le plus concret du terme : équations, schémas, graphiques). Quant au linguistique (ou discursif), il se dédouble lui-même en deux types "d'énoncés»: du plus prouvé (axiomes, postulats, théorèmes, empruntés à d'autres discours, mais aussi clichés et formules figées, le pré-construit), donc une certaine forme d'intertextualité tout de même, et du " personnel » : le discours de l'énonciateur, dont la présence se fait plus ou moins sentir, des énoncés le plus objectifs (définition, loi, démonstration) aux plus périphériques comme la présentation de la problématique ou l'avant-propos, les commentaires, les rappels historiques, les anecdotes éventuelles, les conclusions. L'inscription du sujet dans le discours scientifique serait un bon thème de recherche, car si «penser c'est peser », selon l'aphorisme de Lord Kelvin, l'imaginaire et l'affectivité de l'individu pèsent aussi de tout leur poids sur sa pensée.

35 10. Si l'on retient le concept de «dominante de niveau» d'expression élaboré par le formalisme slave, on peut sans trop de risque affirmer que parmi les niveaux lexical, graphique (mise en page ou " lay-out », schémas) et syntaxique (les seuls pertinents dans le discours scientifique où phonologie et prosodie ne jouent aucun rôle), c'est le niveau lexical qui domine. Ainsi Bally déclare que la syntaxe, entendue comme les procédés grammaticaux de nature exclusivement logique (qu'il classe parmi les « moyens indirects

ASp, 15-18 | 1997 
d'expression ») ne joue aucun rôle discriminatoire en discours scientifique car «il n'y a guère de syntaxe ou de construction propres à la langue scientifique » (t.1., p. 250) et il est vrai que l'unité syntaxique de ce discours qui évite en général les phrases complexes est la structure de base du langage courant: SN R SP car c'est elle qui se rapproche le plus de la formule, objet de la quête scientifique par excellence. Toutefois, la syntaxe mérite tout de même l'attention du stylisticien car l'articulation du linguistique (du discursif) sur le formel pourrait bien avoir des incidences sur ce niveau.

Bally note encore que les langues scientifique et littéraire modifient la langue courante mais pas dans le même sens ni pour les mêmes raisons. La première, tout en y puisant son vocabulaire (que Bally distingue de la terminologie), tend à la rendre aussi impersonnelle et abstraite que possible alors que la seconde veut l'accommoder à une pensée personnelle, affective et esthétique (t.1. 249). L'innovation est réduite à la stricte nécessité. Les mots sont puisés dans la langue courante, parmi les termes les plus propres à exprimer le contenu intellectuel. On parlera par exemple de "siccité » plutôt que de " sécheresse » (trop connotatif ou évocateur). Les néologismes, remarque-t-il, ne font pas problème, hormis un effort de mémorisation... et de lisibilité, notamment orale, serait-on tenté d'ajouter, en songeant par exemple à des termes comme "tetrahydroborate » ou autres «hexamethyleneetetramine $»^{27}$, qui n'ont rien à envier a " antidisestablishmentarianism ».

La double toile de fond étant posée, on peut maintenant tenter de répondre à la question (aux questions) posée(s) par ce Colloque: "Quelle stylistique pour le discours scientifique?».

\section{Stylistique(s) et discours scientifique}

Pour reprendre la distinction langue/discours, si l'on admet l'hypothèse de l'existence d'une langue scientifique commune aux différents secteurs du domaine, l'une des premières tâches de la stylistique, en collaboration avec les linguistes (et les scientifiques, évidemment) serait la description de cette langue, de son lexique ${ }^{28}$, de sa grammaire, de sa rhétorique. La démarche heuristique serait nécessairement une procédure d'extraction: il s'agirait de dégager de la "langue commune " tous les procédés d'expression sinon propres au domaine (rappelons-nous le délicat problème des frontières) du moins privilégiés par lui.

En dehors du lexique spécialisé et savant (de la terminologie scientifique), il faudrait recenser les termes de la langue courante qui ont changé de sens ${ }^{29}$, sans oublier les réajustements périodiques que suppose l'évolution de la «langue» qui s'enrichit progressivement, entre autres acquisitions, de termes scientifiques, d'où les actualisations de plus en plus fréquentes (car le « progrès » s'accélère de jour en jour) des dictionnaires et encyclopédies.

Seconde tâche, dans le prolongement de cette réflexion sur le lexique, l'élaboration d'un métalangage stylistique propre au langage scientifique. L'application à un objet scientifique de concepts forgés pour l'analyse littéraire fait évidemment problème, par exemple les notions centrales de "norme", "forme", "dominante», " registre», « convergence stylistique », «style individuel », « cohérence » et « cohésion» textuelles. Certaines sont clairement non pertinentes ( registre», par exemple), pour d'autres, il 
s'agit moins de changements profonds de signification que de perspective (glissement d'un sémème ${ }^{30}$ à l'autre), et surtout d'enjeux.

41 Ainsi, traditionnellement, pour la stylistique littéraire, la "norme » c'était le « langage ordinaire $»^{31}$, lui-même plus ou moins confondu avec le langage scientifique, tous deux considérés comme des instruments de communication logique et de connaissance objective $^{32}$. Images, «beautés du style» ou «bonheurs d'expression» (comme on disait) relevaient de l'écriture littéraire ${ }^{33}$, considérée comme une déviation par rapport à cette norme. « Normaliser » un discours scientifique peut signifier des choses opposées selon le point de vue adopté : ou bien, c'est le débarrasser des ornements du langage (si on oppose prose littéraire/prose scientifique) ou bien, au risque de déconstruire le discours, c'est supprimer ou remplacer tous les termes techniques et les procédés formulaires (pour le rapprocher du langage ordinaire, à des fins de vulgarisation par exemple), enfin, démarche inverse, c'est ré-introduire la terminologie savante et les formules qui manqueraient dans le texte un peu naif d'un débutant pour le rendre conforme aux exigences du genre (production savante), bref à sa « norme » interne.

Le terme de «forme", d'ailleurs également polysémique en littérature, peut désigner la forme d'une expression linguistique ponctuelle (un mot, une image, une formule), renvoyer à un croquis ou une figure géométrique, où désigner l'ensemble du texte. On se souvient que pour Bally le langage scientifique se confond avec son contenu intellectuel. On retrouve peut-être ici l'analogue de la "forme-sens ", chère aux stylisticiens monistes, selon lesquels forme et contenu sont indissociables (le modèle parfait étant le discours poétique), le moindre changement au plan de l'expression entraînant un changement de contenu. Pourtant, la dichotomie forme/fond est traditionnellement la marque même du langage scientifique (et positiviste) car ce n'est pas la forme de l'expression qui compte mais la vérité objective du contenu : dire « cet objet X coûte 100 francs » au lieu de vaut ou est vendu, n'a aucune importance en arithmétique car c'est l'équivalence logique qui compte, sauf que le rédacteur de l'énoncé aura tendance à choisir le terme le plus neutre possible, de façon précisément à ne pas attirer l'attention sur la forme (à éviter la fonction poétique). En théorie, l'énoncé scientifique (si l'on peut ainsi généraliser) admettrait donc la synonymie ou la paraphrase (quid de la démonstration ?), bref les variantes d'expression - domaine privilégié de la stylistique - avec, mutatis mutandis, les mêmes réserves qu'en littérature, car dans les deux cas (et plus encore dans le domaine scientifique) il y a des limites à la liberté d'expression. Pour reprendre l'exemple cidessus, des énoncés du type "l'objet X va chercher dans les 100 fr chez le marchand », ou encore, «le marchand m'a taxé de 100 balles pour cet article» sont hautement improbables dans des manuels scolaires "standard", aussi improbables que "bleus, d'amour, yeux, vos, marquise, etc. » dans une déclaration d'amour, dans la vie comme au théâtre.

La notion de "dominante ", dont il a été question, est d'un intérêt limité et très général dans le discours scientifique «idéal» (pur, désincarné), puisqu'elle ne concerne que les niveaux d'expression et un type d'énoncé : la démonstration, centre et pièce maitresse du discours. Contrairement à ce qui se passe en littérature (par exemple une image dominante ou obsessionnelle) elle ne peut jamais remettre en question la vérité objective et participer à la production d'un sens qui échapperait aux lois universelles.

Même remarque pour la "convergence stylistique", génératrice de faits stylistiques complexes fondés sur l'interaction des multiples niveaux d'expression ${ }^{34}$. C'est la « convergence stylistique » qui donne au texte littéraire, une épaisseur, un volume, par le 
jeu combiné des redondances ou des tensions aux niveaux du lexique, de la syntaxe, de la phonologie, du rythme et de la prosodie. Comment trouverait-elle place dans un texte scientifique dont la démarche est strictement linéaire, plate, progressive et cumulative plutôt que répétitive? Il ne viendrait pas à l'esprit, par exemple, d'analyser le rôle de l'allitération dans le discours scientifique, encore moins ses rapports avec le niveau sémantique. Il a été dit que la description en stylistique (la recherche des faits pertinents), nécessairement sélective, est toujours plus ou moins orientée par les réactions personnelles (décrire c'est choisir, choisir c'est interpréter), lesquelles sont elles-mêmes en partie déterminées par des facteurs externes : type ou genre de discours, mode esthétique, connaissance de l'auteur, préjugés culturels, etc. Il va de soi que le lecteur n'aborde pas un texte scientifique (ou un article de journal) de la même façon qu'il lit un texte littéraire: les "horizons d'attente» («expectations, frames of reference ») sont très différents et la sensibilité esthétique (hormis les lieux communs sur la beauté des raisonnements abstraits) est en sommeil dans le premier cas. Le texte scientifique peut multiplier les schèmes allitérants, ceux-ci seront considérés comme aléatoires, fruit du fonctionnement mécanique de la langue, sauf fantaisie ou facétie de l'auteur, dont il faudrait apporter la preuve ${ }^{35}$.

45 Quant à la notion de "style individuel », elle mérite nuance dans le domaine scientifique où la recherche est souvent plus collective que dans le domaine littéraire, au sein de laboratoires regroupant des équipes de chercheurs participant aux mêmes expériences, parlant plus ou moins le même langage et publiant des ouvrages en commun. Par exemple le «style » du pseudo Nicolas Bourbaki est celui de l'équipe de la rue d'Ulm (Cartan, Chevalley, Delsarte, Dieudonné, Weil) gravitant autour de ce personnage fictif, Euclide des temps modernes, qui révolutionna le discours mathématique avec ses Éléments de mathématique en s'appuyant notamment sur la logique formelle et la théorie des ensembles et dont l'ambition principale était de réaliser la grande synthèse, de ramener les mathématiques à la mathématique, d'élaborer en d'autres termes un langage unique, accessible même au lecteur non initié ${ }^{36}$.

L'approche stylistique du discours scientifique idéal suppose donc, en premier lieu, l'élaboration d'un métalangage adapté à son objet et quelques choix méthodologiques reposant sur des considérations (peut-être idéologiques, après tout) quant à la nature même de ce discours. Cela fait, rien ne s'oppose, si le discours scientifique (pour l'instant toujours considéré comme "idéal») est une pratique discursive parmi d'autres à une application des trois approches stylisticiennes rappelées plus haut.

47 L'analyse stylistique peut s'appliquer à différents niveaux du texte isolé, avec deux objectifs principaux: exploration des rapports complexes (complémentarité ou opposition ; imitation ou déviation) entre langue ordinaire et langue scientifique, et des liens qui subsistent entre la perception sensorielle et l'imagination, d'une part, et la conceptualisation ou le raisonnement logique, d'autre part. Selon une démarche descriptive comparable à celle de l'analyse littéraire, elle tâchera de rendre compte, par exemple, des faits suivants :

48 - changements de sens des lexèmes, à deux niveaux : en comparant langue ordinaire et langue scientifique ${ }^{37}$, mais aussi à l'intérieur de la seconde, en explorant l'évolution des acceptions (dénotations, mais aussi connotations qui peuvent jouer quand même un rôle) d'un même terme en fonction de l'avancement des connaissances, dans le sens, par exemple, d'une plus grande précision des concepts. 
49 - recherche de l'unité de base du discours scientifique ; analyse structurale de la phrase simple et/ou complexe, enchaînement des propositions grammaticales; configuration du paragraphe conceptuel, etc.

50 - contraction de la syntaxe (énoncés elliptiques); rôle des parenthèses ; préférence pour telle ou telle modalité (assertive, impérative, interrogative) ${ }^{38}$; rôle de la voix passive, de la ponctuation, des anaphoriques, en particulier des pronoms personnels dont, semble-til, le discours scientifique use avec parcimonie, préférant répéter les concepts et les notions (Annexe, 8) ; rôle aussi des déictiques, par exemple la première personne plurielle (nous, we) qui ne fonctionne sans doute pas toujours de la même façon qu'en discours littéraire, où elle prend souvent place dans une stratégie de manipulation du lecteur, inconcevable en discours scientifique. Le scientifique est par définition ou convention un «reliable author ».

51 - rôle des formules figées liées à tel ou tel raisonnement, des présupposés (on imagine qu'il n'y, a pas de « sous-entendus » en discours mathématique ou, si le terme est utilisé, le sens n'est pas le même qu'en pragmatique [Ducrot 1972]).

52 - articulation du discursif sur le formulaire (les équations, schémas, etc.).

53 - recours au langage profane à des fins pédagogiques. Ce qui permet, par exemple à un Valéry de déclarer, quelque part, que le génie de Newton a consisté à dire que la lune tombe alors que tout le monde voit bien qu'elle ne tombe pas.

54 - aspects typographiques de la présentation : disposition sur la page (« lay-out »), division en chapitres, sections, sous-sections avec leur numérotation (caractère fragmenté du texte scientifique), retraits de paragraphes, mise en relief des schémas, utilisation des polices de caractères, etc. Ces aspects concrets de l'écriture scientifique sont particulièrement importants dans les manuels scolaires, une façon sans doute d'aider la mémoire et de corriger l'abstraction.

55 - mais aussi dimension esthétique éventuelle, dont l'école bourbakiste nous apprend, et ce fut une surprise pour moi, qu'elle a aussi son rôle à jouer dans le discours scientifique.

56 Quant aux méthodes et protocoles d'analyse, si le discours scientifique est par excellence le «texte de la vérité » et du discours sérieux et réussi, les propositions de la science relèvent d'une sémantique vériconditionnelle, celles de la littérature d'une sémantique intentionnelle. À ce titre, la théorie des actes de parole et celle des maximes conversationnelles de Grice sont pertinentes pour son étude, puisque ce discours est par définition « coopératif», libre de toute « implicature » ou « flouting " : l'information doit être aussi complète que possible (maxime de quantité), vraie en tous points (maxime de qualité), pertinente, claire et concise (maxime de manière... ou de style). Le discours scientifique relève donc, entre autres pratiques, d'une stylistique pragmatique (Jaubert 1990 : ch. viii).

57 Après la description minutieuse du texte isolé, de son matériau linguistique et formel, de son fonctionnement en contexte, l'analyse stylistique peut élargir son enquête à une série aussi étendue que possible d'autres textes d'une même variété (secteur scientifique) puis de variétés différentes. Cette description des procédés d'écriture pour chaque discours permettrait sans doute de repérer des constantes, des traits de style, de vérifier par exemple, si la voix passive caractérise bien certaines variétés de discours scientifique Crystal \& Davy $(1969: 55,251)$, etc. ; bref, en dégageant des " profils stylistiques » ${ }^{39}$, elle poserait les jalons d'une véritable étude de style (différentielle et comparative) du type stylistique des genres ${ }^{40}$, qui apporterait à son tour sa contribution à l'établissement d'une 
typologie des discours scientifiques, selon les conditions d'énonciation (discours oral, discours écrit; discours de vulgarisation, discours savant), le mode de communication (note ponctuelle, rapport détaillé d'expérience, essai, compte rendu critique, ouvrage théorique, manuel scolaire), le domaine d'application (mathématique, chimie, physique, géologie, astronomie, biologie, etc.) et à l'intérieur d'une "langue de spécialité », une typologie des énoncés (postulats, théorèmes, équations, démonstrations, graphiques, schémas). Elle pourrait aussi tenter de répondre à la question de savoir s'il existe une écriture scientifique féminine.

L'analyse rhétorique (en diachronie comme en synchronie) comprend la technique d'argumentation (inventio et dispositio) et les ornements du style (elocutio), notamment les images ${ }^{41}$. Il convient tout d'abord de souligner un fait historique. À l'origine, il n'y a pas de rhétorique du langage scientifique, car il ne relève pas de l'art oratoire. La rhétorique couvrait traditionnellement trois types de discours :

1. - le délibératif, qui concernait le discours politique

2. - le judiciaire (forensic) : discours juridique (plaidoirie ou accusation)

3. - le démonstratif: discours d'éloge ou l'hommage funèbre.

Quant aux modes de persuasion, ils étaient aussi au nombre de trois : pathos (on joue sur les émotions), ethos (on invoque le sens moral du locuteur: un bon orateur doit être irréprochable dans sa conduite), logos (on s'adresse à la raison, à l'intelligence). C'est évidemment le dernier mode qui est, en principe, seul sollicité dans le discours scientifique.

La rhétorique, appliquée au discours scientifique, concerne en priorité la technique de démonstration et d'argumentation, marquant les différences entre l'argumentation en langue quotidienne et en logique pure (Plantin 1990 : 38-44). Renouant avec la logique (à laquelle on l'a jadis opposée ${ }^{42}$ ), elle analyse, par exemple, les différents «styles de raisonnement scientifique» (Hacking, cité par Molino 1994), décrit leur différentes formes et fonctionnement et leur adéquation au propos.

61 Mais quelle place pour la rhétorique des images en discours scientifique? Quelques remarques préalables s'imposent. Historiquement, la rhétorique des figures reste tributaire d'une stylistique de l'écart, de la déviation par rapport à une norme : cette norme serait une langue neutre, blanche, sans relief, sans image, bref un mythe. En revanche, quand un mathématicien oppose langage mathématique et langage ordinaire, le sens de ce dernier s'inverse. Pour le mathématicien, la langue ordinaire est marquée par la subjectivité, la figuralité, et c'est le discours mathématique qui serait ce langage strictement neutre et intellectuel, en d'autres termes, cette norme indéfinissable que postule le littéraire ${ }^{43}$. La conséquence de ces renversements de sens et de perspective est que les concepts habituels utilisés en stylistique littéraire ainsi que la terminologie rhétorique traditionnelle risquent d'être en partie inadéquats pour le discours scientifique. L'utilisation du terme "symbole " en mathématique est un exemple trop évident ${ }^{44}$. Autre exemple : le terme "abus de langage », que j'ai rencontré sous la plume d'un mathématicien, ne désignait pas la catachrèse de la rhétorique classique (dont la métaphore est un exemple) mais renvoyait à une expression littérale jugée inadéquate dans le contexte car elle ne correspondait pas à l'idée pure qu'il voulait exprimer.

Le langage scientifique, qui se radicalise surtout au XVIIe siècle avec l'avènement de la nouvelle physique, repose massivement sur la logique formelle, or le logique rejette, en principe, l'analogique donc la figure, trope ou non trope (comparaison n'est pas raison). 
Mais aucun discours, si rationnel soit-il, n'échappe au langage imagé. Tout d'abord, l'analogique est au cœur même de la pensée autant et peut-être même plus que le logique, comme l'ont très bien montré Lakoff et Johnson $(1980)^{45}$. Deuxièmement, aucun discours n'est jamais parfaitement homogène. Langue ordinaire et langue technique se mêlent inévitablement, sauf à réduire le discours scientifique à une suite de formules algébriques, d'algorithmes ou d'équations ${ }^{46}$. Les images interviennent donc à deux niveaux, comme trace de la présence du langage ordinaire, dans les introductions, passages de transition, éventuellement dans les conclusions, ou comme partie intégrante du raisonnement scientifique. La langue de la physique nucléaire, par exemple, est pleine de métaphores. De même, si la linguistique est une science, on sait combien son métalangage est traversé par une foule d'images empruntées non seulement aux sciences exactes mais aussi à la nature, aux activités humaines et au monde des objets ${ }^{47}$.

La différence essentielle avec le langage littéraire est à la fois quantitative (fréquence basse), qualitative (choix des images) et fonctionnelle : la figure est mise au service sinon du logique, du moins du référentiel. C'est pourquoi les images les plus efficaces se transmettent et se répètent, sans gêne pour l'auteur et le lecteur, car elles sont en quelque sorte banalisées, lexicalisées, aussi indispensables à la démonstration que les valeurs mesurables, les équations ou les modèles de raisonnement.

Un dernier point: il ne faudrait pas oublier que les mathématiques, et plus encore les sciences de la nature, relèvent d'une stylistique des images au sens le plus littéral, visuel et graphique du terme, que la révolution informatique est d'ailleurs en train de bouleverser (images virtuelles). L'insertion d'une image ou plutôt d'une « figure » (c'est le terme le plus souvent retenu en science) ou encore d'une photo dans un texte écrit et les rapports entre les «deux énoncés " supposent toute une série de choix stylistiques qui dépendent de stratégies de communication ou d'édition et qui méritent des analyses approfondies.

Je voudrais maintenant revenir sur un point important. L'allusion faite plus haut à l'énonciation et les dernières remarques sur la place des images dans le discours scientifique nous ramènent à la notion ambiguë de « texte scientifique idéal ». Tel qu'il a été évoqué (plutôt que défini), il désignait surtout le discours de spécialiste s'adressant à des spécialistes. Or le « discours scientifique » doit être mis à la portée de tout un chacun et le « discours idéal » sera celui qui assurera, pour un auditoire donné, le succès de la communication, de l'initiation la plus élémentaire au domaine au discours le plus savant. La stylistique, en collaboration étroite avec la pédagogie, la didactique, la psychologie, les praticiens des langues de spécialité, mais aussi les maisons d'édition, peut ainsi participer utilement à l'élaboration des manuels scolaires et de toutes les formes de diffusion de la connaissance scientifique.

Dans un autre ordre d'idée, moins pragmatique, on peut dire qu'il n'y a pas en réalité de "discours scientifique idéal », comme l'avait déjà remarqué Bally ${ }^{48}$. Si le discours logique, artificiel, désincarné, de la science est traversé par la langue, qui résiste, entre par effraction ou s'insinue à chaque instant dans le propos, il est aussi, à des degrés divers certes, hanté par la mythologie personnelle du locuteur, par ses habitudes linguistiques, bref par son style, et ce style menace, comme la langue ordinaire, l'intégrité du discours. Et nous retrouvons ici cet élément perturbateur que nous avions chassé au début.

L'étude du style, (ré)entendu maintenant comme la trace de l'individuel dans le général, retrouve ainsi sa place mais les enjeux ont changé: il ne s'agit plus d'élaborer une modélisation du discours mais de traquer toutes les formes de modalisation qui contrarie 
ce projet. La recherche du style individuel n'a d'autre intérêt que de repérer dans l'énoncé supposé objectif la trace de l'intrus, du parasite, c'est-à-dire d'établir la faillite du discours scientifique, sa non-représentativité, alors que l'enquête stylistique en littérature est tout le contraire: en dégageant les traits stylistiques, en signalant la présence de l'individuel et de l'unique dans l'énoncé, elle tend à rendre compte de la « littérarité » ou de l'exemplarité du discours, du moins si l'unique est bien le critère de la littérarité, selon une certaine idéologie. La différence, à ce niveau de la problématique, est d'importance. En littérature, la stylistique de caractérisation individuelle fournit en même temps la clé du mystère, permet la conquête de l'objet ; en science, elle se sépare radicalement du contenu. Dans le premier cas, individuel et général se confondent, dans le second, ils s'opposent. Du strict point de vue des enjeux scientifiques, une telle stylistique n'est donc pas pertinente pour le discours scientifique. En revanche, elle retrouve une certaine légitimité dans une perspective didactique et pédagogique s'il s'agit de dénoncer le mauvais exemple auprès d'un groupe d'apprenants et de leur enseigner les vertus du style impersonnel, si je puis risquer cet oxymore, un style (ou une absence de style, ou un " plain style ») qui s'apprend donc dans les écoles. S'il y a une stylistique du discours scientifique, elle ne peut être que normative, prescriptive et donc proscriptive, générale, à l'image de la rhétorique antique.

\section{Conclusion}

La place de la stylistique (des stylistiques) dans le discours scientifique est évidemment plus limitée que celle qu'elle occupe dans son domaine privilégié, le texte littéraire, dans la mesure où, pour beaucoup, le discours scientifique est le lieu même du non-style, mais elle est loin d'être négligeable.

À un niveau très élémentaire (pédagogique, didactique), le discours scientifique pose évidemment des problèmes de style puisqu'il s'agit, pour l'élève ou le savant en herbe, d'acquérir un langage spécialisé qui soit aussi adapté que possible à son objet tout en évitant l'excès d'abstraction ou de technicité (jargon) qui nuirait à la communication ${ }^{49}$, bref il s'agit d'être « scientifiquement correct ».

Plus généralement, le discours scientifique, comme toute autre activité langagière, peut se prêter à toutes les pratiques stylisticiennes (étude de style, analyse stylistique, analyse rhétorique) et sous-pratiques (stylistique des genres, des thèmes, des mots, des formes, des intentions, des effets, stylistique énonciative, etc.), le choix de la pratique dépendant - ici comme en littérature - à la fois de la nature de l'objet (du domaine) analysé, et des questions qu'on se pose à son sujet. Par exemple, la vérité scientifique n'étant produite ni par l'auteur ni par le lecteur ${ }^{50}$, la «stylistique des intentions» et la «stylistique des effets» ne sont pas ou sont peu pertinentes en discours scientifique, leur degré de pertinence étant directement proportionnel au degré de déviation de tel ou tel discours par rapport au schéma idéal (au modèle platonicien).

71 Comme il ne semble pas qu'il existe vraiment une grammaire élaborée du discours scientifique, la stylistique du discours scientifique est surtout une stylistique de l'argumentation et une stylistique lexicale: néologismes, formation des mots ${ }^{51}$, motsvalises («blendings» ou portmanteau-words), rapports entre vocabulaire courant et vocabulaire technique, utilisation d'hyperonymes ${ }^{52}$ (de sens générique), etc. Aussi libre que possible des connotations, des images, de l'implicite et de la polysémie, cette 
stylistique des mots se prête plus facilement que le langage littéraire à l'étude statistique (stylo-statistique).

La démarche comparative de l'étude de style peut apporter sa contribution à la définition du profil du langage scientifique et des énoncés divers qui entrent dans la composition du texte scientifique, dans le cadre général de l'établissement d'une typologie des discours (aspect synchronique), ainsi qu'à l'histoire de son développement au cours des âges (aspect diachronique).

Elle pourrait apporter des éléments de réponse à la question indirectement soulevée par l'expérience Bourbaki : "Y a-t-il une esthétique du discours scientifique?», en dehors évidemment des lieux communs sur le frémissement métaphysique dont le corps est saisi devant une belle équation ou un raisonnement impeccable ou des extases keatsiennes sur la beauté de la vérité et la vérité de la beauté.

La stylistique de la traduction ("traductologie», dit-on maintenant), ou stylistique contrastive (terme assez vague) ou stylistique comparée des langues ouvre un champ d'investigation très stimulant qui dépasse le simple passage mécanique (si la chose a un sens) d'une langue de départ à une langue d'arrivée. Elle peut nous en apprendre beaucoup sur l'incidence des différences de mentalité et de culture sur la constitution de ce discours supposé « universel » qu'est le discours scientifique. Peut-être y a-t-il, après tout, un style français de la démonstration mathématique et un style anglais.

Dans tous les cas, l'application de la stylistique au discours scientifique pose l'épineux problème du métalangage, ne serait-ce que pour éviter des télescopages lexicaux, la discipline ayant souvent emprunté sa terminologie à la science. Qu'on songe, par exemple, à des termes comme "structure», "valeur», "fonction», "principe d'équivalence». Parler (si cela est concevable) de la valeur (stylistique) d'une valeur (mathématique), de la fonction d'une fonction... ce n'est pas du bon « style»!

Une ultime remarque en guise de conclusion: la tenue d'un tel Colloque aura au moins permis de ré-affirmer l'existence et la pérennité de la stylistique, discipline que quelques esprits chagrins ne cessent d'enterrer périodiquement, faisant ainsi la preuve de sa capacité à renaître sans cesse de ses cendres.

\section{BIBLIOGRAPHIE}

Bally, Ch. 1909 [1951]. Traité de stylistique française, 3e édit. (deux tomes). Paris : Klincksieck.

Bourbaki, N. 1934. Éléments de mathématique, Théorie des Ensembles. Paris : Hermann, Fasc. XVII.

Bulletin (New series) of the American Mathematical Society 22/1, January 1990.

Black, M. 1962. Models and Metaphors. New York : Ithaca.

Crystal, D. \& D. Davy. 1969. Investigating English Style. Londres : Longman.

Ducrot, O. 1972. Dire et ne pas dire. Paris : Hermann.

Epstein, E.L. 1978. Language and Style (New Accents). Londres : Methuen. 
Fish, S. 1980. Is There a Text in this Class. The Authority of Interpretive Communities. Cambridge, MA : Harvard University Press.

Jaubert, A. 1990. La lecture pragmatique. Paris : Hachette.

Kilmister, C.W. 1973. General Theory of Relativity. Oxford : Pergamon.

Lakoff, G. et M. Johnson. 1980. Metaphors We Live by. Chicago, IL : University of Chicago Press.

Mathis, G. 1994. « Fait stylistique relationnel ». Bulletin de la Société de Stylistique anglaise 15, 4-30.

Molino, J. 1979. « Métaphores, modèles et analogies dans les sciences ». Langages 54, La

Métaphore, 83-125.

Molino, J. 1994. « Pour une théorie sémiologique du style ». In Molinié, G. et P. Cahné (dir.). Qu'est-ce que le style, Actes du Colloque de 1991 sur ce thème, Paris 4 Sorbonne. Paris : Presses Universitaires de France, 213-261.

Perec, G. 1991. « De Iaculatione Tomatonis (in cantatricem) ». Faude, 1987 (Trad. française dans Cantatrix sopranica L. et autres écrits scientifiques. 1991. Paris : Seuil).

Petit, M. 1995. « De la pertinence du 'stylistic profiling' pour l'étude du discours de spécialité ». Bulletin de la Société de Stylistique anglaise 16, 67-80.

Plantin, Ch. 1990. Essais sur l'argumentation. Paris : Kimé.

Richards, I.A. 1924 [1967]. Principles of Literary Criticism. Londres : Routledge \& Kegan Paul.

Schlanger, J. 1983. L'invention intellectuelle. Paris : Fayard.

Wales, K. 1989. A Dictionary of Stylistics. Londres : Longman.

\section{ANNEXES}

\section{Corpus et éléments de commentaire}

\section{Exemple 1}

« On peut aussi y écouter des CD audio, visionner des films au format DVD ou encore lire CD-Rom et DVD-Rom. Un système de téléphone mobile mains libres, via un GMS intégré et entièrement piloté du micro-ordinateur (à base de Pentium MMx cadencé à $200 \mathrm{MHz}$ ), complète l'équipement. Ah, dernier détail, ce terminal, équipé d'un fax-modem, permet la communication par courrier électronique ainsi que la connexion à Internet... » (Le Nouvel Observateur 1723, $1997: 26$. $)^{53}$

Dans cet énoncé de cinq lignes, on ne relève pas moins de sept sigles, une dizaine de termes techniques qui ne sont passés dans la langue courante (c'est-à-dire non seulement connus, mais compris) que pour les initiés car l'énoncé ci-dessus, tiré d'une revue grand public, aurait tout aussi bien sa place dans une revue spécialisée.

\section{Exemple 2}

« The electrons were thought to be stuck in this charged ball like raisins in a cake. » ( Bulletin (New Series) of the American Mathematical Society 22/1, January $1990^{54}$ : 3.)

\section{Exemple 3}


The inequality ... can be stated poetically as follows. An electron is like a rubber ball, or a fluid, with an energy density proportional to ... It costs energy to squeeze it and this accounts for the stability of atoms. (Bulletin: 13.)

Le terme « poetically » est utilisé ici de façon sommaire comme synonyme de " métaphoriquement » ou plutôt « d'analogiquement », puisqu'il s'agit en fait d'une (double) comparaison. Le littéraire, personnage délicat et sensible, peut s'interroger sur le degré de poéticité du premier comparant « rubber ball », qu'il prend en pleine figure, et s'offenser de la collocation oxymorique « stated poetically ", car il sait depuis la Defence of Poesy de Sidney («the poet nothing affirmeth and therefore never lieth ») et I.A. Richards (" poetry is pseudo-statement ») que la poésie n'affirme ni ne « déclare » rien. Un comparant donc, très « domestique » (comme dans l'exemple précédent) et prosaïque, qui semble suggérer plusieurs choses : ou bien le locuteur n'a aucun sens poétique ou bien il inclut le « poétique » (tel qu'il le définit sans trop d'inspiration) dans le langage ordinaire, dont son discours prétend se démarquer (l'énoncé « poétique » est en italiques dans le texte, mais d'autres énoncés mathématiques, considérés comme importants, le sont aussi), auquel cas on trouve ici confirmation du renversement des perspectives, puisque pour le littéraire et le poète, le langage ordinaire est plus ou moins synonyme de langage scientifique, mais il est vrai que le poète n'est pas à une métaphore près.

Fait plus intéressant, la collocation de deux comparants ( rubber ball » et « fluid », plus technique) appartenant à deux mondes différents et produisant une analogie hétérogène. S'ouvre ici une possible piste de recherche stylistique : les analogies complexes dans le discours scientifique.

\section{Exemple 4}

«Using poetic license again, we can say :

A large atom is like a galaxy. It has a small, high density, energetic core in which most of the electrons are to be found and whose size decreases with $\mathrm{z}$ like $\mathrm{z}-1 / 3$. The electronelectron electrostatic repulsion always manages to push a few electrons out to a distance of order one which is roughly independent of $\mathrm{z}$ : this is the radius one observes if one tries to «touch » an atom. » (Bulletin : 21).

- Confirmation des préjugés quant au langage poétique, avec cette différence : le comparant (scientifique) est choisi dans le même champ disciplinaire, la " poéticité » tenant ici non à la nature de l'image, mais au procédé formel (analogique).

- Contraste entre la phrase courte d'ouverture et la phrase complexe qui suit, essentiellement descriptive (factuelle), fortement articulée par ses connecteurs logiques.

- Dans cette même phrase, exemple de groupe nominal canonique, avec ce syntagme adjectif étendu « small, high density, energetic... ", très factuel, décrivant les propriétés physiques de l'objet, l'équivalent en quelque sorte du nom savant composé. La prémodification (adjectifs devant le nom) serait, en effet, un trait de style du discours scientifique (mais c'est aussi la place normale en « langue ordinaire ») et la postposition des adjectifs, un signe de littérarité ou de poéticité. Autre question : l'ordre croissant des volumes syllabiques $(1,4,4)$ dans ce groupe est-il stylistiquement pertinent?

- De même la répétition de certains mots (« electron ») pas plus que l'allitération finale «tries/touch/atom » ne semblent devoir concerner le stylisticien, sauf, encore une fois, à 
s'interroger sur les caprices du langage (même scientifique), interrogations qui risquent fort de déboucher sur des apories.

\section{Exemple 5}

« The energy in a smallish stone, $N=1023$ would be that of a hydrogen bomb [D]. Such 'matter' would be unpleasant stuff to have lying around the house. ».

- Mélange de discours scientifique (y compris formulaire) et de discours ordinaire qui vise à frapper l'imagination (populaire), exploitant donc à la fois le logos (on s'adresse à la raison) et le pathos (émotion) de la rhétorique d'argumentation.

\section{Exemple 6}

« In brief, the imposition of the Pauli exclusion principle raises E. The miracle is that it raises $\mathrm{E}$ enough so that stability of the second kind holds ». (Bulletin : 15)

- « miracle » : modulation évaluative signalant l'irruption du locuteur.

\section{Exemple 7}

«So, from the equation of motion, the acceleration produced in a body at a particular point of the gravitational field is the same whatever the mass of that body. To put it in an inexact but easily remembered form : « all bodies fall equally fast » (Kilmister 1973:4).

- Exemple typique de modalisation et de juxtaposition de deux énoncés, soit un discours scientifique idéal et sa « traduction » en langue ordinaire, donc « inexacte », les guillemets indiquant que le locuteur ne prend pas en charge cette énonciation. Cette contamination du discours scientifique est rendue nécessaire par l'impossibilité pratique (pragmatique) de s'en tenir au pur langage de l'abstraction, en raison des limites de l'esprit humain et des modes de fonctionnement de la mémoire. Le discours scientifique a besoin du discours profane pour être efficace et cette navette entre les deux types de discours est directement fonction de la situation d'énonciation. On peut aisément poser qu'elle sera d'autant plus fréquente en communication orale et en présence d'un auditoire jeune et/ou non spécialiste. L'ouvrage d'où cet extrait est tiré est un « textbook » destiné à des étudiants (plus précisément des « undergraduates »).

- Question : comme le locuteur recherche un procédé mnémotechnique (et la poésie à l'origine est bien cela), l'allitération « fall/fast » redevient sans doute pertinente, mais elle reste, de toute façon, inscrite dans un énoncé déviant par rapport au discours scientifique. Poursuivant la lecture, on s'aperçoit (voir exemple suivant) que la citation revient, cette fois sans guillemets, comme intégrée à ce discours. En fait, il n'en est rien car les guillemets sont remplacés par la formule déclarative « as we have said » qui fonctionne comme trace de la modalisation, car malgré le « we » trompeur, le savant continue de ne pas prendre en charge (ou en tout cas pas totalement) cette énonciation. Enfin, l'énoncé « inexact » revient une troisième fois, quelques lignes plus loin, cette fois, sans précaution oratoire, présenté comme un fait : « ...on the fact that all bodies fall equally fast » (5). Mais a-t-il vraiment changé de « valeur » ou de statut? Probablement pas car il fait partie en réalité de la séquence d'énoncés qui a commencé avec la première occurrence fortement modalisée, s'est poursuivie avec la seconde qui l'était déjà moins, pour aboutir à la dernière, faussement objectivée. Bref, il en va dans le discours scientifique comme dans le discours littéraire, l'analyse isolée d'un énoncé (ou d'une image) présente parfois des risques de « misreading ». 


\section{Exemple 8}

« ...by the postulation of gravitational forces, and says that, as long as gravitational forces are allowed ... so that a particle released from rest in this frame remains at rest in the frame, since, as we have said, all particles fall equally fast ... using as an inertial frame a frame fixed to... » (Kilmister $1973: 4-5$ )

- Rejet du pronom anaphorique avec la répétition de « gravitational forces » et de «frame » (par deux fois).

\section{Exemple 9}

«... has really only been chosen as a historical - or possibly geographical -accident » (Kilmister $1973: 5$ )

- Exemple de modalisation adverbiale, de « hedging » (cf. Lakoff et Johnson 1980 et Wales 1989 : 215-6).

\section{Exemple 10}

a) If the two points are coincident, say R1 = R2 = 0, we have a "bomb"...

What prevents this from happening?

Answer: the nucleus-nucleus repulsive energy. (Kilmister 1973 : 37)

b) What mechanism is available...?

Answer: The quantum mechanical kinetic energy of fermions. (Kilmister $1973: 39$ )

- Énoncé typique de la forme schématique que tend à prendre le discours scientifique : un « jeu » de questions/réponses rappelant une équation mathématique. Notons que la distribution parallèle des énoncés, avec la répétition de certains lexèmes (« What », " Answer ») constitue en rhétorique une anaphore. En pragmatique anglo-saxonne, ce type d'énoncés liés porte divers noms : « adjacency pair », « tied pair », « illocutionary sequel » (cf. Wales, $1991: 10)$.

- Les guillemets pour « bomb », contrairement à ce qui se passe dans le discours poétique, signalent l'intrusion du langage métaphorique.

\section{Exemple 11}

Extraits de Cantatrix Sopranica, de G. Perec :

a) "As observed at the turn of the century by MARKS \& SPENCER (1899), who first named the "yelling reaction" (YR), the striking effects of tomato throwing on Sopranoes have been extensively described...In their henceforth late twenties' classical demonstrations, CHOU \& LAI (1927 a, b, c, 1928 a,b, 1929 a, 1930) have ruled out the hypothesis of a pure facio-facial nociceptive reflex that has been advanced for many years by a number of authors (MACE \& DOYNE, 1912; PAYRE \& TAIRNELLE, 1916; SORNETTE \& BILLEVAYZE, 1925). [...] Recent observations by UNSOFORT \& TCHETERA pointing out that "the more you throw tomatoes on Sopranoes, the more they yell" and comparative studies dealing with the gasp-reaction (OTIS \& PIFRE, 1964), hiccup (CARPENTIER \& FIALIP, 1964), cat purring (REMMERS \& GAUTIER, 1972), HM reflex (VINCENT et al., 1976), ventriloquy (MCCULLOCH et al., 1964), shriek, scream, shrill and other hysterical reactions (STURM \& DRANG 1973 ) have led to the steady assumption of a positive feedback organization of 
the YR based upon a semilinear quadristable multi-switching interdigitation of neuronal sub-networks functioning en désordre (BEULOTT et al., 1974)."

- Marqueurs scientifiques : le point, exhaustif (on imagine) sur la recherche antérieure; abondance de références bibliographiques datées, de termes techniques, dont composés d'origine lexicale savante et lourds pré-modificateurs devant le mot-noyau (« semilinear quadristable multi-switching interdigitation »); citation genre théorème ; utilisation de sigles.

- Traitement parodique et ironique : caractère facétieux des références bibliographiques avec jeux de mots, certains hétéroglossiques (« UNSOFORT \& TCHETERA » pour « and so forth, etc. ", par exemple); double sens de « striking » (syllepse) ; inversion des guillemets pour " yelling reaction"; caractère tautologique (évident) du « théorème » (« the more you throw tomatoes.... »); effet ironique de la séquence en decrescendo dans la parenthèse CHOU \& LAI (a,b,c, puis a,b, puis a) puis rien ; pour un effet ironique différent, voir l'emploi des minuscules dans la parenthèse sur Giscard d'Estaing, dans l'extrait suivant, bel exemple de rupture d'une norme, donc de fait stylistique, selon Riffaterre)

b) «Préparation

Experiments were carried out on 107 female healthy Sopranoes (Cantatrix Sopranica L.) furnished by the CONSERVATOIRE NATIONAL DE MUSIQUE, and weighing 94-124 kg (mean weight : $101 \mathrm{~kg}$ ). Holothane anesthesia was utilized during the course of tracheotomy, fixation in the Horsley-Clarke, and major operative procedures. 5\% procaine was injected into skin margins and pressure points. Animals were then immobilized with gallamine triethyiodide $(40 \mathrm{mg} / \mathrm{kg} / \mathrm{hr}$ ) and normocapnia was maintained by appropriate artificial ventilation. Spinal cord transections were performed at L3/T2 levels, thus eliminating blood pressure variations and adrenaline secretion induced by tomato throwing (Giscard d'Estaing, 1974).

The fact that animals were not suffering from pain was shown by their constant smiling throughout the experiments. Internal temperature was maintained at $38^{\circ} \mathrm{C} \pm 4^{\circ} \mathrm{F}$ by means of three electrically driven boiling kettles. »

- Marqueurs scientifiques : abondance des mesures (« penser c'est peser »); fréquence de la voix passive, plus impersonnelle (agent anonyme) et description détaillée du protocole expérimental (comme dans l'exemple suivant).

c) «Stimulation

Tomatoes (Tomato rungisia vulgaris) were thrown by an automatic tomatothrower (WAIT \& SEE, 1972) monitored by an all-purpose laboratory computer DID/92/85/P/331) operated on-line. Repetitive throwing allowed up to 9 projections per sec, thus mimicking the physiological conditions encountered by Sopranoes and other Singers on stage (TEBALDI, 1953). Care was taken to avoid missed projections on upper and/or lower limbs, trunks and buttocks. Only tomatoes affecting faces and necks were taken into account.

Control experiments were made with other projectiles, such as apple cores, cabbage runts, hats, roses, pumpkins, bullets and ketchup (HEINZ, 1952). » 


\section{NOTES}

1. Même Aristote, avec lequel naît la méthode scientifique, déclare: «La physique et les mathématiques ne doivent être regardées que comme des parties de la philosophie » ( Métaphysique, IX.iv.).

2. Procédé qu'on retrouve par exemple au 12e siècle dans la rhétorique des "questions » qui permettent de retrouver les «lieux» de l'argumentation sous la forme de cet hexamètre quis, quid, ubi, quibus, auxiliis, cur, quomodo, quando, où sont exploitées allitérations, assonances et rimes internes.

3. Elle n'est qu'un « artefact » dont la nature est d'ailleurs loin d'être clairement définie : s'agit-il de discours oraux ou de discours écrits, ou des deux ? D'autre part, la langue est en perpétuelle mutation et lorsqu'on parle de « langue », il s'agit, le plus souvent d'un « état de langue », lequel est loin d'être parfaitement homogène car rien ni personne n'échappe jamais à son histoire. Enfin, « langue » se confond souvent avec « langage ordinaire », c'est-à-dire un type de discours, celui de la communication quotidienne.

4. Diversement appelée « register» (Firth) ou " province » (Crystal/Davy) ou «field » par les théoriciens anglais.

5. Toutefois, l'emploi du terme est trompeur car les revues que j'ai pu consulter montrent assez que dans la plupart des cas il s'agit surtout de "nuls avancés ", à moins que je n'aie déjà pris un retard considérable en la matière. Ce décalage lexical entre langue ordinaire et langue spécialisée est en soi révélateur des problèmes que pose le développement ultra rapide de la science et de la technologie dans le monde moderne.

6. Voir le corpus en Annexe (exemple 1), un échantillon de cette langue commune spécialisée.

7. La montée en puissance, pour utiliser un cliché branché (... sur le discours scientifique) des surdoués dont on parle beaucoup en ce moment, est peut-être le signe avant-coureur des nouveaux clivages sociaux qui se préparent, dans un monde où l'aristocratie du cerveau remplacera la noblesse de sang.

8. La profession de foi qui suit n'engage évidemment que moi.

9. Observons que la multiplication des champs de recherche depuis un demi-siècle a eu pour conséquence la confiscation de territoires jadis occupés par la stylistique, rebaptisés pragmatique, sémiotique littéraire, poétique, etc., si bien que beaucoup font de la stylistique sans le savoir... ou sans vouloir l'admettre, car le mot n'est, paraît-il, plus à la mode. Pourtant le vénérable ancêtre fait de la résistance et continue d'affirmer son existence dans des composés comme "stylo-linguistique », «stylistique pragmatique », "sémiostylistique », «stylistique actantielle » (branche de la narratologie), « stylistique énonciative », etc.

10. "Style is meaning, nothing else is ", continuait d'affirmer Stanley Fish, le "one-time» partisan d'une «stylistique affective » (très différente de celle de Bally), dans un entretien privé à Duke University (été 1991).

11. En effet, quand on croit identifier un «style individuel » (unique, inimitable), on reconnaît d'abord quelqu'un que l'on connaît mieux qu'un autre, c'est tout. Seule une analyse différentielle élaborée (exhaustive, donc impossible) pourrait espérer établir cette " unicité ». Voir infra sur « étude de style ».

12. Le concept de "pertinence stylistique » est évidemment à la fois capital et délicat en stylistique. Disons qu'il se définit en grande partie en fonction des questions que l'on pose au texte. Chaque analyse stylistique doit donc s'attacher à bien définir ses objectifs et ses critères de sélection des faits retenus.

13. Est-il besoin de préciser que l'analyse stylistique n'a pas pour objectif de démontrer ou prouver la validité de tel ou tel modèle linguistique. D'autre part, elle peut varier ses modèles 
selon la nature de l'objet analysé. Un certain éclectisme théorique et méthodologique n'est donc pas forcément un défaut en stylistique.

14. Il est révélateur que des linguistes de plus en plus nombreux s'intéressent à la stylistique et à la rhétorique, malgré leur réticence parfois à le reconnaître.

15. Pour des raisons théoriques, pratiques et pédagogiques qu'il serait trop long d'expliquer. Mais on se souvient du fiasco d'une certaine analyse structurale des Chats de Baudelaire par LeviStrauss et Jakobson où l'abondance des faits décrits finissait par engendrer la lassitude et le scepticisme, tandis que l'arbre cachait la forêt.

16. Je préfère finalement cette expression banale à «stylistique fonctionnelle », car la notion de «fonction" intervient aussi dans l'Étude de style, dans la mesure où c'est souvent moins le procédé linguistique en lui-même qui fonde le trait de style que l'usage particulier qui en est fait, sinon le trait de style se réduirait à une simple empreinte anthropométrique qui ne livrerait de l'homme que... le bout des doigts, et la stylistique à une recherche en paternité.

17. «Style as perceptive strategy », selon E.L. Epstein (1978, chapitre 1), dont la «stylistique des effets » de Riffaterre est une variante.

18. Il existe des dictionnaires de faits rhétoriques mais pas de dictionnaire de faits stylistiques dont la production est en théorie illimitée. Ajoutons que le terme « analyse rhétorique » est luimême ambigu car il renvoie à au moins trois pratiques très différentes : ou bien on se donne à l'avance une figure précise (par exemple la métaphore) et on en fait une description formelle et/ ou son histoire (et l'on reste dans le domaine strict de la Rhétorique), ou bien on l'étudie, en synchronie ou en diachronie, dans tel ou tel corpus, pour retracer son évolution ou dégager des dominantes, voire des obsessions (cf. la psychocritique de J.P. Weber ou de Ch. Mauron), auquel cas l'analyse rhétorique peut recouper les deux autres pratiques, mais elle reste une étude partielle et orientée ; ou bien encore, on appellera « analyse rhétorique » cette partie de l'analyse stylistique portant sur les procédés répertoriés par les traités classiques, dans un texte donné.

19. Les mathématiques sont à la science ce que la poésie est à la littérature, deux paradigmes qui ne sont pas ou n'ont pas toujours été si éloignés qu'on veuille bien le dire, d'ailleurs, car il y a aussi de la poésie dans les mathématiques et de la mathématique dans la poésie, comme dans la musique. Le terme technique de "numbers ", parfois synonyme de "verse " ou "poetry » est d'une certaine manière révélateur de leurs rapports. On peut aussi invoquer le rôle important de la numérologie en poésie, dans ses fonctions symboliques et structurales, dans la poésie de la Renaissance, celui des analogies scientifiques dans la poésie métaphysique ou, plus près de nous, les expériences mi-sérieuses mi ludiques de l'Oulipo, dont l'entreprise, on s'en souvient, fut inspirée par les Fondements de la Géométrie, de David Hilbert.

20. Bien que Bally pratique une stylistique de la langue et non du discours, on trouvera de nombreuses remarques intéressantes sur le discours scientifique dans son Traité de Stylistique française.

21. Je m'en tiens strictement au «texte» en laissant de côté le «paratexte » (éditorial ou autorial), deux dimensions de l'écriture que la réflexion postmoderne réunit maintenant dans la notion globale de «textualité » qui tend à remplacer «texte» et «structure». Comme cette problématique intéresse surtout le domaine littéraire, elle ne nous concernera pas ici.

22. Citons cette définition du groupe Bourbaki, placée, notons-le au passage, sous le signe de la modalité conditionnelle: «On arrive ainsi à la conclusion qu'un texte mathématique suffisamment explicite pourrait être exprimé dans une langue conventionnelle ne comportant qu'un petit nombre de "mots" invariables assemblés suivant une syntaxe qui consisterait en un petit nombre de règles inviolables : un tel texte est dit formalisé » $(1934: 1)$.

23. Pour Bally, le discours scientifique est une activité purement intellectuelle (ni affectivité, ni intention esthétique). C'est la langue des idées, la littérature, celle des sentiments (1909 [1951], t. $1: 245)$ : «la langue scientifique est pour nous l'ensemble des moyens d'expression par lesquels l'esprit cherche à décrire la réalité ou à démontrer la vérité, c.-à-d. que le langage scientifique se 
confond, théoriquement, avec le mode d'expression intellectuel tout entier. Un texte scientifique "authentique" révèle toujours, par le choix des mots et le tour des phrases, le besoin de ne montrer que la face objective des choses, et l'on sent que, chez le vrai savant, ce mode d'expression résulte d'un effort à la fois incessant et inconscient » (118).

24. Respectivement, termes dialectaux, anachronismes, changements de niveaux de langue ou de registre, irruption d'un "style» ou d'un type de discours inattendu (le poétique dans le scientifique, par exemple).

25. Ce qui ne veut pas dire évidemment que l'émotion, l'imagination, l'intuition, l'affectif, voire l'esthétique n'ont pas leur place dans le domaine scientifique. Une démonstration peut être belle et ce n'est pas un hasard si les sciences ont souvent fasciné plus d'un poète.

26. La notion de "vérité » en discours scientifique n'est en fait pas si évidente que cela. Dans ce domaine comme ailleurs, une vérité chasse l'autre. Les «vérités " d'un Euclide sont contestées par Lobatchewski et Riemann, celles de Newton par Einstein. En brûlant du plomb et en le pesant (donc en pensant!), Lavoisier réfute la théorie chimique du phlogistique. D'autre part, la réflexion moderne remet en question l'existence « des absolus mathématiques » et les concepts mêmes de « vérité » ou de «fausseté » perdent de leur pertinence. On ne pose plus telle ou telle proposition mathématique comme absolument vraie mais comme "nécessaire », à l'intérieur d'un système donné. L'histoire de la science, comme celle de la poésie, est traversée de révolutions.

27. Notons toutefois que la terminologie savante est moins cryptique qu'il n'y paraisse à première vue : elle est souvent l'image linguistique de son objet, énumérant sous forme plus ou moins abrégée ses différents composants. Cela dit, la terminologie savante mérite peut-être (si cela n'a pas déjà été fait) une analyse morphologique détaillée.

28. Il ne s'agit pas évidemment d'une encyclopédie ou d'un inventaire des «objets» de connaissance, avec leurs noms savants, qui n'aurait d'intérêt que pour le chercheur scientifique. 29. "Changé de sens" est sans doute impropre car bien souvent, ces acceptions sont en puissance dans la langue (par l'étymologie, par exemple), mais elles ont été déplacées, enfouies par l'usage. La science, tournée vers l'avenir, est aussi un retour aux origines, à l'étymon des mots comme des choses, d'où sa dimension poétique.

30. Faut-il rappeler que « sémème » est le «sens » que prend un lexème en contexte : «table » (de la loi, de multiplication, de restaurant...), par exemple?

31. On suppose que l'expression a un sens, tout en se souvenant de la question « How ordinary is ordinary language? », de Stanley Fish : qu'il est opportun de citer : «Ordinary language» is one of a number of terms used to designate a kind of language that «merely» presents or mirrors facts independently of any consideration of value, interest, perspective, purpose and so on. Other such terms as «literal language», «scientific language», «propositional language», «logical language», «denotative language», «neutral language», «mathematical language», «serious (as opposed to fictional) language», «non-metaphorical language», representational language», «messagebearing language», «referential language», «descriptive language», and «objective language». Whatever the term, the claim is always the same: it is possible to specify a level at which language correlates with the objective world... » (1980: 97).

32. L'identification discours prosaïque/discours scientifique est en effet aussi répandue chez les théoriciens que celle de littérature/poésie.

33. Un Dumarsais, l'auteur du Traité des Tropes, relevait déjà au XVIIIe siècle, donc bien avant Lakoff et Johnson, l'importance des images dans la langue « commune ", déclarant qu'il se fait en un jour à la Halle plus d'images qu'en huit jours à l'Académie. Certes, mais peut-être faudrait-il distinguer entre « langue ordinaire » et « langue populaire » (il y a une littérature populaire).

34. Pour une pratique stylistique fondée sur le concept de "convergence ", voir la thèse de Michael Riffaterre sur Le Style des Pléiades de Gobineau(Paris : Droz, 1957). 
35. De même, si le style c'est l'homme (formule souvent mal interprétée), ce qui intéresse au premier chef le «scientifique » (auteur ou lecteur) ce n'est pas la fiche d'identité, la "photo » d'Einstein, mais plutôt le photon, si je puis me permettre cette paronomase, et la formule $\mathrm{E}=$ mc2.

36. On se prend à rêver à un Bourbaki linguiste qui nous rendrait le même service mais je n'ai pas l'impression qu'on en prenne vraiment le chemin. Cela dit, le groupe Bourbaki intéresse au premier chef la stylistique car l'un des traits dominants de cette école, c'est son travail sur le vocabulaire. Il est puisé dans le langage courant mais le sens des mots est complètement changé. Ce sens est livré par les axiomes et les définitions antérieures. Ces scientifiques s'approprient ainsi le langage quotidien, au sens le plus fort du verbe. D'autre part, les mots - et ceci est tout de même surprenant - sont choisis en fonction de la commodité, de l'élégance des sons, ou pour faire un jeu de mots.

37. Peut-être convient-il de nuancer l'affirmation (globalement vraie) de Bally, selon laquelle, dans le discours scientifique, contrairement au discours littéraire, les modifications du vocabulaire courant portent sur les mots, pas sur les sens (1909 [1951] t.1. : 244-5).

38. Sans parler de la modalisation adverbiale de prudence, par exemple "possibly" (voir Annexe, 9) car le discours scientifique qui ne détient pas la vérité absolue mêle affirmations et hypothèses.

39. Traduction imparfaite du «stylistic profiling» de Crystal (1990). Voir pour la méthode, l'article de Petit (1995).

40. Différente évidemment de la stylistique des genres littéraires (plus diversifiés, sans doute). Par exemple, la distinction classique (et médiévale) entre les trois degrés du style, connue sous le nom de «roue de Virgile» (style élevé, moyen ou bas, selon les genres, triade calquée sur la hiérarchie de la Cité idéale platonicienne) n'est pas pertinente en langage mathématique où l'idéologie ne joue aucun rôle (en principe).

41. Rappelons qu'avec le développement de l'imprimerie et la confiscation du « rhétorique » par le "littéraire ", ces deux aspects de la rhétorique générale classique se sont séparés pour ne laisser qu'une « rhétorique restreinte ", limitée aux figures (Fontanier) et même à une vingtaine de tropes (Dumarsais).

42. En particulier à l'époque de la Renaissance... et de la rhétorique restreinte (à l'elocutio, aux ornements du langage), où deux images figuraient cette opposition: le poing serré pour la logique, la main ouverte pour la rhétorique.

43. C'est pourquoi, lorsqu'il s'agit de définir la spécificité du langage poétique, on l'oppose souvent, par commodité, au langage mathématique, censé représenter la langue standard dans son état le plus pur. Pour une opposition poésie/science, voir I.A. Richards (1924 [1967]).

44. Mais rappelons qu'un même symbole, par exemple $a$ peut ne pas renvoyer au même contenu d'un raisonnement à l'autre dans le même texte.

45. Molino (1979) rappelle que le rôle de l'analogie dans l'invention scientifique a été souligné depuis longtemps, notamment par Max Black, le théoricien de la métaphore interactionnelle, dans Models and Metaphors (1962). Pour le rôle des métaphores dans le discours mathématique, on peut aussi se reporter à l'ouvrage de Judith Schlanger (1983).

46. Ainsi voit-on un «Bourbaki » légitimer finalement l'emploi de la rhétorique, après avoir rejeté l'analogique du discours formalisé : «...contiendront des commentaires destinés à rendre plus claire la marche des idées, au besoin par un appel à l'intuition du lecteur; l'emploi des ressources de la rhétorique devient dès lors légitime, pourvu que demeure inchangée la possibilité de formaliser le texte » $(1934: 4)$.

47. On peut citer les arbres de la grammaire générative, les patates de la théorie des ensembles, les mots-noyaux de certaines grammaires, le triangle de la signification, le carré sémiotique, les cames de Culioli, la différentielle signifiante de Kristeva, les catalyseurs du Barthes narratologue s'inspirant des formalistes russes, etc. 
48. « ...le côté personnel et affectif de la pensée qui tend toujours et malgré tout à s'infiltrer dans l'expression de l'idée pure» (1909 [1951] t.1 : 118), y compris sous la forme d'images car le langage scientifique est finalement beaucoup plus imagé qu'on ne le pense.

49. D'où les conseils en matière d'écriture prodigués par certaines revues savantes, par exemple le Handbook for Chemical Society of Authors (1961) cité par Crystal et Davy (1969 : 251). Sans parler des problèmes de lisibilité que posent certains monstres lexicaux, de type joycien, comme «tetramethycyclooctate». On serait tenté d'ajouter «...et de mémorisation», mais le signe scientifique est moins arbitraire qu'il n'y parait puisque le signifiant est souvent la transcription linguistique stricte de la composition interne (chimique, physique) de l'objet.

50. Selon les théories post-modernes de la « textualité », le lecteur est « producteur » du texte.

51. Selon K. Wales (1989: 220), le discours scientifique se caractériserait par la fréquence des mots hybrides combinant plusieurs langues, comme «wash-able " (saxon + français ou latin), «television » (grec + latin)

52. C'est-à-dire de lexèmes de sens générique : «animal » pour «chien », comme on parle de "sujets» pour les cobayes dans les expériences. Pour un emploi ironique, voir le pastiche de Perec, Annexe, 11, b, (« Animals » pour « sopranos »).

53. À propos d'un nouveau modèle de véhicule automobile, façon James Bond.

54. Ci-après Bulletin.

\section{RÉSUMÉS}

Cette communication, après avoir présenté la pluralité des pratiques de la stylistique (étude de style, analyse de style, analyse rhétorique) en liaison avec les conceptions du style et dégagé les éléments fondamentaux de la caractérisation du discours scientifique, pose les conditions épistémologiques et méthodologiques d'une application spécifique de la stylistique à l'étude du discours scientifique. Elle en illustre la pratique par quelques exemples en annexe.

This paper presents the diversity of stylistic practice (study of style, stylistic analysis, rhetorical analysis) together with the various conceptions of style, defines the fundamental characteristics of scientific discourse and formulates some of the epistemological and methodological conditions for a specific stylistic approach to scientific discourse. A few examples of such an approach are annexed.

\section{INDEX}

Mots-clés : analyse rhétorique, analyse stylistique, discours scientifique, langue de spécialité, style (étude de), stylistique, texte

Keywords : LSP, rhetoric, rhetorical analysis, scientific discourse, study of style, style, stylistic analysis, stylistics, text 


\section{AUTEUR}

\section{GILLES MATHIS}

Gilles Mathis, agrégé d'anglais, docteur d'État, est professeur de littérature (domaines poétique et dramatique) et de stylistique anglaises à l'Université de Provence (Aix-Marseille 1) et Président de la Société de stylistique anglaise. Il est l'auteur d'une thèse intitulée « Analyse stylistique du Paradis Perdu : l'univers poétique, échos et correspondances ", soutenue en 1979. Il a écrit une quarantaine d'articles sur les problèmes théoriques de la stylistique littéraire et sur Milton, Donne, Herbert, Vaughan, Wordsworth, Hopkins, Yeats, Shakespeare, etc. Il travaille actuellement sur un manuel de stylistique littéraire (théorie et pratiques). 